\title{
DIVISION ET EXTENSION DANS DES CLASSES DE CARLEMAN DE FONCTIONS HOLOMORPHES
}

\author{
VINCENT THILLIEZ \\ CNRS - URA 751, Bât. M2, Mathématiques \\ Université des Sciences et Technologies de Lille \\ 59655 Villeneuve d'Ascq Cedex, France \\ E-mail: Vincent.Thilliez@univ-lille1.fr
}

Dédié au Professeur Stanistaw Lojasiewicz

\begin{abstract}
Let $\Omega$ be a bounded pseudoconvex domain in $\mathbb{C}^{n}$ with $C^{1}$ boundary and let $X$ be a complete intersection submanifold of $\Omega$, defined by holomorphic functions $v_{1}, \ldots, v_{p}$ $(1 \leq p \leq n-1)$ smooth up to $\partial \Omega$. We give sufficient conditions ensuring that a function $f$ holomorphic in $X$ (resp. in $\Omega$, vanishing on $X$ ), and smooth up to the boundary, extends to a function $g$ holomorphic in $\Omega$ and belonging to a given strongly non-quasianalytic Carleman class $\left\{l ! M_{l}\right\}$ in $\bar{\Omega}$ (resp. satisfies $f=v_{1} f_{1}+\ldots+v_{p} f_{p}$ with $f_{1}, \ldots, f_{p}$ holomorphic in $\Omega$ and $\left\{l ! M_{l}\right\}$ regular in $\bar{\Omega}$ ). The essential assumption is that $f$ and $v_{1}, \ldots, v_{p}$ belong to some (maybe smaller) Carleman class $\left\{l ! M_{l}^{-}\right\}$, where the sequences $M^{-}$and $M$ are precisely related by geometric conditions on $X$ and $\Omega$.
\end{abstract}

Introduction. Soit $\Omega$ un ouvert borné, pseudoconvexe à bord lisse, dans $\mathbb{C}^{n}$, et soit $A^{\infty}(\bar{\Omega})$ l'algèbre des fonctions holomorphes dans $\Omega$ et $C^{\infty}$ jusqu'au bord de $\Omega$ (autrement dit, admettant un prolongement $C^{\infty}$ dans $\left.\mathbb{C}^{n}\right)$. On considère $v_{1}, \ldots, v_{p}(1 \leq p \leq n-1)$ des fonctions de $A^{\infty}(\bar{\Omega})$ ainsi prolongées à $\mathbb{C}^{n}$ et on pose $\tilde{X}=\left\{v_{1}=\ldots=v_{p}=0\right\}$, $X=\tilde{X} \cap \bar{\Omega}$.

Lorsque l'on a $\partial v_{1} \wedge \ldots \wedge \partial v_{p} \neq 0$ sur $X \cap \partial \Omega$ et que les ensembles $\bar{X} \backslash \bar{\Omega}$ et $\bar{\Omega}$ sont régulièrement situés au sens de Łojasiewicz $[\mathrm{M}]$, on connait les propriétés suivantes:

(a) Toute fonction $f$ de $A^{\infty}(\bar{\Omega})$ qui s'annule sur $X$ vérifie la propriété de division $f=v_{1} f_{1}+\ldots+v_{p} f_{p}$ avec $f_{j} \in A^{\infty}(\bar{\Omega})$ pour $j=1, \ldots, p$.

1991 Mathematics Subject Classification: Primary 32E25, 32E35; Secondary 46E99.

Received by the editors: November 2, 1996.

The paper is in final form and no version of it will be published elsewhere. 
(b) Toute fonction $f$ qui, en un sens convenable, est $C^{\infty}$ sur $X$ et holomorphe dans l'intérieur relatif de $X$ dans $\tilde{X}$, s'obtient par restriction à $X$ d'une fonction $g$ de $A^{\infty}(\bar{\Omega})$.

Il s'agit là de résultats d'E. Amar [A1]. On se reportera également à P. de Bartolomeis \& G. Tomassini [DBT], R. Gay \& A. Sebbar [GS], ainsi qu'aux références citées dans ces travaux.

Soit à présent $M=\left(M_{l}\right)_{l \geq 0}$ une suite croissante de réels positifs, logarithmiquement convexe. Disons qu'une fonction $h$ de classe $C^{\infty}$ au voisinage d'une partie $Y$ de $\mathbb{C}^{n}$ satisfait des estimations Carleman $C_{M}$ sur $Y$ s'il existe une constante $C$ telle que les dérivées de $h$ à tout ordre $l$ soient bornées par $C^{l+1} l ! M_{l}$ sur $Y$. Comme on le sait, la suite $M$ mesure, en un certain sens, le défaut d'analyticité de $h$ sur $Y$.

Dans ce travail, on exploite les résultats de [Th1] pour répondre aux questions suivantes. On se place dans le cadre des résultats (a) et (b) rappelés précédemment et on suppose que la suite $M$ est "fortement régulière" (voir $\S 1$, par exemple $M_{l}=l !^{\alpha}(\log l)^{\beta l}$, $\alpha>0, \beta \in \mathbb{R})$. Peut-on alors, à partir de la donnée de $M, \Omega$ et $\tilde{X}$, déterminer une suite $M^{-}$telle que dans les résultats (a) et (b), une hypothèse additionnelle de régularité $C_{M^{-}}$, portant sur $f$ et sur les $v_{j}$ dans $\bar{\Omega}$, implique que les fonctions $f_{j}$ et $g$ aient la régularité $C_{M}$ sur $\bar{\Omega}$ ? Dans l'affirmative, quelles sont les propriétés de $M^{-}$?

Ce problème se ramène à l'étude de l'idéal engendré par les $v_{j}$ sur la classe des fonctions de $A^{\infty}(\bar{\Omega})$ qui vérifient des estimations $C_{M}$ sur $\bar{\Omega}$. Après l'introduction d'outils techniques aux $\S 1$ et $\S 2$, les résultats obtenus sont décrits au $\S 3$. Les démonstrations font l'objet des $\S 4$ et $\S 5$.

La méthode suivie fait appel à deux étapes: une première étape consiste à établir des versions locales des résultats précités. Pour cela, on s'inspire en particulier de constructions faites par E. Amar [A1] dans le cas de $A^{\infty}(\bar{\Omega})$. La seconde étape consiste à globaliser les résultats via des procédés cohomologiques; ici la proposition 6.1 de [GS] joue un rôle crucial. Bien entendu, il est également nécessaire de disposer de solutions du $\bar{\partial}$ à régularité Carleman: ces solutions sont fournies par [CC2] lorsque l'ouvert $\Omega$ possède une "bonne" base de voisinages pseudoconvexes.

Tout au long de l'article, et en contraste avec le cas de $A^{\infty}(\bar{\Omega})$ où l'aspect quantitatif est occulté, il est essentiel de contrôler très précisément les "pertes de régularité" Carleman liées à la propriété de situation régulière des ensembles $\bar{X} \backslash \bar{\Omega}$ et $\bar{\Omega}$. Ceci nécessite l'introduction de procédés spécifiques: en particulier, le théorème de recollement de jets ultradifférentiables et la notion de situation régulière raffinée de [Th1] sont essentiels.

\section{Résultats préliminaires}

DÉfinition 1.1. Soit $M=\left(M_{l}\right)_{l \geq 0}$ une suite de réels positifs. On dit que la suite $M$ est fortement régulière lorsqu'elle vérifie les conditions suivantes:

(1.1.1) Les suites $M$ et $\left(M_{l+1} / M_{l}\right)_{l \geq 0}$ sont croissantes.

(1.1.2) Il existe une constante $A_{1} \geq 1$ telle que l'on ait, pour tout $l$,

$$
M_{l} \leq A_{1}^{l} M_{j} M_{l-j} \text { pour } 0 \leq j \leq l .
$$


(1.1.3) Il existe une constante $A_{2} \geq 1$ telle que l'on ait, pour tout $l$,

$$
\sum_{j \geq l} \frac{M_{j}}{(j+1) M_{j+1}} \leq A_{2} \frac{M_{l}}{M_{l+1}} .
$$

On note $\mathcal{S}_{\text {fr }}$ l'ensemble des suites fortement régulières.

On définit une relation d'équivalence $\sim \operatorname{sur} \mathcal{S}_{\text {fr }}$ en disant que l'on a $M \sim M^{\prime}$ si et seulement si il existe une constante $C$, avec $C \geq 1$, telle que l'on ait $C^{-(l+1)} M_{l} \leq M_{l}^{\prime} \leq$ $C^{l+1} M_{l}$ pour tout entier $l$.

On désigne par $\mathcal{F}$ l'ensemble des fonctions $\varphi: \mathbb{R}_{+} \longrightarrow \mathbb{R}_{+}$croissantes au voisinage de 0 dans $\mathbb{R}_{+}$et telles que $\varphi(0)=0$. On définit aussi une relation d'équivalence $\simeq$ sur $\mathcal{F}$ en disant que l'on a $\varphi \simeq \psi$ si et seulement si il existe des constantes $b, c$, avec $0<b<1$, $0<c<1$, telles que l'on ait $b \varphi(c t) \leq \psi(t) \leq b^{-1} \varphi\left(c^{-1} t\right)$ pour $t \rightarrow 0$.

1.2. Fonctions $h_{M}$. Soit $M$ une suite fortement régulière. Pour tout entier $l$, on pose

$$
h_{M}(t)=\inf _{j \geq 0} t^{j} M_{j} \quad \text { pour } \quad t \in \mathbb{R}_{+} .
$$

La fonction $h_{M}$ est continue, croissante et on a $h_{M}(0)=0$ et $h_{M}(t)=M_{0}$ pour $t \geq 1$. De plus, si on pose $N_{l}=\operatorname{Sup}_{t>0} t^{-l} h_{M}(t)$, on a alors $N \sim M$ en vertu de la condition de convexité logarithmique (1.1.1). La donnée de $h_{M}$ détermine donc $M$ dans $\mathcal{S}_{\mathrm{fr}} / \sim$. Comme autre conséquence, étant données deux suites $M$ et $M^{\prime}$ de $\mathcal{S}_{\mathrm{fr}}$, l'existence de constantes $b$ et $c$ avec $0<b<1,0<c<1$, telles que l'on ait $b h_{M}(c t) \leq h_{M^{\prime}}(t)$ pour $t \longrightarrow 0$, équivaut à l'existence d'une constante $C \geq 1$ telle que l'on ait $M_{l} \leq C^{l+1} M_{l}^{\prime}$ pour tout entier $l$. En particulier, on a $M \sim M^{\prime}$ si et seulement si on a $h_{M} \simeq h_{M^{\prime}}$ dans $\mathcal{F}$. Compte tenu de ces remarques, on confondra souvent, dans le reste de ce travail, les suites $M$ de $\mathcal{S}_{\text {fr }}$ et leurs classes d'équivalence modulo $\sim$.

EXEMPLE. Soient $\alpha$ et $\beta$ réels, avec $\alpha>0$. La suite $M$ donnée par $M_{l}=l !^{\alpha}(\log l)^{\beta l}$ pour $l \geq 1$ est fortement régulière et on a $h_{M}(t) \simeq \exp \left(-t^{-1 / \alpha}(\log (1 / t))^{-\beta / \alpha}\right)$.

DÉfinition 1.3. Soit $\theta$ un élément de $\mathcal{F}$. On dira que la fonction $\theta$ est fortement admissible si elle vérifie les conditions suivantes :

(1.3.1) Elle est continue, strictement croissante, au voisinage de 0 dans $\mathbb{R}_{+}$.

(1.3.2) La fonction $t \longrightarrow \theta(t) / t$ est croissante au voisinage de 0 dans $\mathbb{R}_{+}$.

(1.3.3) Il existe un réel $q>1$ tel que la fonction $t \longrightarrow \theta(t) / t^{q}$ soit décroissante au voisinage de 0 dans $\mathbb{R}_{+}$.

EXEMPLE. Un exemple standard de fonction fortement admissible est donné par $\theta(t)=$ $t^{\mu}\left(\log \left(1+\frac{1}{t}\right)\right)^{-\nu}$, où $\mu$ et $\nu$ sont deux réels, avec $\mu \geq 1$ et $\nu \geq 0$.

La définition 1.4 ci-après illustre l'usage que l'on fera, dans ce travail, des fonctions fortement admissibles. On désigne par $d$ la distance euclidienne.

DÉfinition 1.4. Soient $Y_{1}$ et $Y_{2}$ deux sous-ensembles fermés de $\mathbb{R}^{n}$ et $\theta$ une fonction fortement admissible. Si on a $Y_{1} \cap Y_{2} \neq \emptyset$, on dit que $Y_{1}$ et $Y_{2}$ sont $\theta$-situés lorsque, quels que soient les sous-ensembles compacts respectifs $E_{1}$ et $E_{2}$ de $Y_{1}$ et $Y_{2}$, il existe une constante $\gamma>0$ et un ouvert $V$ contenant $E_{1} \cup E_{2}$ tels que l'on ait

$$
d\left(x, E_{1}\right)+d\left(x, E_{2}\right) \geq \gamma \theta\left(d\left(x, E_{1} \cap E_{2}\right)\right)
$$


pour tout $x$ de $V$. Si on a $Y_{1} \cap Y_{2}=\emptyset$, on convient aussi de dire que $Y_{1}$ et $Y_{2}$ sont $\theta$-situés avec $\theta(t)=t$ (autrement dit: $i d$-situés).

Remarque 1.5. Dans la définition précédente, il est possible d'employer des fonctions admissibles plus générales, voir [Th1] et [Th2]. Ici, il sera techniquement plus simple de se limiter aux fonctions fortement admissibles définies en 1.3. Il s'agit d'une restriction minime, voir les points 1.6 et 1.7 de [Th2].

Il convient par ailleurs de remarquer que, compte tenu de (1.3.3), la $\theta$-situation définie en 1.4 implique la situation régulière de Łojasiewicz [M]. Elle en est une forme précisée.

On rappelle maintenant une construction faite dans la proposition 2.2 de [Th1]. Elle jouera un rôle essentiel aux $\S 3$ et $\S 4$.

Proposition 1.6. Soit $\theta$ une fonction fortement admissible. Alors, quel que soit $M$ dans $\mathcal{S}_{\mathrm{fr}} / \sim$, il existe un unique $M^{(\theta)}$ dans $\mathcal{S}_{\mathrm{fr}} / \sim$ tel que l'on ait

$$
h_{M} \simeq h_{M^{(\theta)}} \circ \theta \text {. }
$$

En outre, $M^{(\theta)}$ est donné explicitement, modulo $\sim$, par

$$
M_{l}^{(\theta)}=\prod_{0 \leq j \leq l-1} m_{j}^{(\theta)} \text { avec } m_{j}^{(\theta)}=\frac{1}{\theta\left(1 / m_{j}\right)}, \quad m_{j}=M_{j+1} / M_{j} .
$$

EXEMPLES.

(i) Pour $\theta(t)=t^{\mu}(\mu \geq 1)$, on a $M_{l}^{(\theta)}=\left(M_{l}\right)^{\mu}$. En particulier, pour $\theta=$ id (identité), on a $M^{(\theta)}=M$.

(ii) Pour $M_{l}=l !^{\alpha}(\log l)^{\beta l}$ et $\theta(t)=t^{\mu}\left(\log \left(1+\frac{1}{t}\right)\right)^{-\nu}$ avec $\alpha>0, \beta \geq 0, \mu \geq 1$ et $\nu \geq 0$, on a $M_{l}^{(\theta)}=l !^{\alpha \mu}(\log l)^{(\beta \mu+\nu) l} \quad($ On vérifie (1.6.1) à l'aide de l'exemple donné en 1.2 , ou bien on utilise (1.6.2)).

On a très facilement la propriété suivante: soient $\theta$ une fonction fortement admissible et $M$ une suite de $\mathcal{S}_{\text {fr }} / \sim$; alors il existe des constantes $q \geq 1$ et $C \geq 1$ telles que l'on ait

$$
C^{-(l+1)} M_{l} \leq M_{l}^{(\theta)} \leq C^{l+1}\left(M_{l}\right)^{q}
$$

pour tout $l$ (c'est une conséquence directe de (1.3.2), (1.3.3) et (1.6.2)). La propriété qui suit est beaucoup moins immédiate.

Proposition 1.7 ([Th2], proposition 1.10). Soit $\theta$ une fonction fortement admissible. Alors l'application $M \longrightarrow M^{(\theta)}$ est une bijection de $\mathcal{S}_{\mathrm{fr}} / \sim$ sur lui-même.

1.8. Notation. Dans toute la suite, si $\theta$ est une fonction fortement admissible donnée et si $M$ est un élément de $\mathcal{S}_{\text {fr }} / \sim$, on notera $M^{-}$l'unique élément de $\mathcal{S}_{\text {fr }} / \sim$ tel que l'on ait $\left(M^{-}\right)^{(\theta)}=M$.

\section{Classes de Carleman}

2.1. Notations. Pour $z=\left(z_{1}, \ldots, z_{n}\right)$ dans $\mathbb{C}^{n}$, on pose $z_{j}=x_{j}+i x_{n+j}(1 \leq j \leq n)$. Pour tout multi-indice $L=\left(l_{1}, \ldots, l_{2 n}\right)$ de $\mathbb{N}^{2 n}$, on note $l$ la longueur $l_{1}+\ldots+l_{2 n}$ de $L$ et $D^{L}$ le monôme de dérivation $\partial^{l} / \partial x_{1}^{l_{1}} \cdots \partial x_{2 n}^{l_{2 n}}$ associé à $L$.

2.2. Classes de Carleman. Soit $M$ une suite fortement régulière. Une fonction $f$ de $C^{\infty}\left(\mathbb{C}^{n}\right)$ sera dite appartenir à la classe de Carleman $C_{M}\left(\mathbb{C}^{n}\right)$ s'il existe une constante 
positive $C$ (dépendant de $f$ ) telle que l'on ait, pour tout multi-indice $L$ de $\mathbb{N}^{2 n}$ et tout $z$ de $\mathbb{C}^{n}$,

$$
\left|D^{L} f(z)\right| \leq C^{l+1} l ! M_{l} .
$$

La classe $C_{M}\left(\mathbb{C}^{n}\right)$ est une algèbre, stable par opérateurs différentiels. Elle est fortement non-quasianalytique $[B]$, en particulier dotée de partitions de l'unité.

Soit à présent $\Omega$ un ouvert borné à bord de classe $C^{1}$ dans $\mathbb{C}^{n}$. On définit la classe de Carleman $C_{M}(\bar{\Omega})$ comme l'algèbre des fonctions $f$ de $C^{\infty}(\bar{\Omega})$ telles que l'on ait (2.2.1) pour tout multi-indice $L$ et tout $z$ de $\bar{\Omega}$, et on définit $A_{M}(\bar{\Omega})$ comme l'algèbre des fonctions de $C_{M}(\bar{\Omega})$ qui sont holomorphes dans $\Omega$. D'après [B], [CC1] (voir aussi le corollaire 3.12 de $[\mathrm{BBMT}])$, pour toute fonction $f$ de $C_{M}(\bar{\Omega})$, il existe une fonction $\tilde{f}$ de $C_{M}\left(\mathbb{C}^{n}\right)$ telle que l'on ait $\left.\tilde{f}\right|_{\bar{\Omega}}=f$; ainsi $A_{M}(\bar{\Omega})$ peut être encore vu comme l'ensemble des fonctions $f$ de $C_{M}\left(\mathbb{C}^{n}\right)$ telles que l'on ait $\bar{\partial} f=0$ sur $\bar{\Omega}$. Plus généralement, si $K$ est un compact de $\mathbb{C}^{n}$, on notera $A_{M}(K)$ l'ensemble des fonctions $f$ de $C_{M}\left(\mathbb{C}^{n}\right)$ telles que $\bar{\partial} f$ s'annule à l'ordre infini sur $K$ (compte tenu des théorèmes d'extension de [B], [CC1], cette notion coïncide avec celle de jet $\bar{\partial}$-plat de classe $C_{M}$ sur $K$ ).

Soit $\mathcal{F}$ un faisceau de germes de fonctions sur $\bar{\Omega}$. On désigne par $\mathcal{F}^{(p, q)}$ le faisceau des germes de $(p, q)$-formes différentielles à coefficients dans $\mathcal{F}$, par $\Gamma(\bar{\Omega}, \mathcal{F})$ l'espace des sections de $\mathcal{F}$ et par $\mathcal{F}_{\zeta}$ la fibre de $\mathcal{F}$ en un point $\zeta$ de $\bar{\Omega}$. On note $C_{M}$ (resp. $A_{M}$ ) le faisceau sur $\bar{\Omega}$ des germes de fonctions de $C_{M}(\bar{\Omega})$ (resp. $A_{M}(\bar{\Omega})$ ) et $\mathcal{O}$ le faisceau usuel sur $\mathbb{C}^{n}$ des germes holomorphes. On identifie $\Gamma\left(\bar{\Omega}, C_{M}\right)$ et $C_{M}(\bar{\Omega})\left(\right.$ resp. $\Gamma\left(\bar{\Omega}, A_{M}\right)$ et $\left.A_{M}(\bar{\Omega})\right)$.

On remarquera que toutes les notions précédentes ne dépendent que de la classe de $M$ modulo $\sim$.

Définition 2.3 [CC2]. Un compact $K$ de $\mathbb{C}^{n}$ est dit 1 -H-convexe s'il existe une constante $c$, avec $0<c<1$, telle que pour tout réel $\delta>0$, assez petit, on puisse trouver un ouvert $\Omega_{\delta}$ pseudoconvexe satisfaisant

$$
\{z: d(z, K)<c \delta\} \subset \Omega_{\delta} \subset\{z: d(z, K)<\delta\} .
$$

\section{EXEMPLES.}

(i) Si $\Omega$ est un ouvert borné à bord $C^{2}$ strictement pseudoconvexe, ou plus généralement $C^{1}$ à fonction définissante plurisousharmonique, alors $\bar{\Omega}$ est 1-H-convexe.

(ii) L'adhérence de tout ouvert $\Omega$ borné pseudoconvexe à bord $C^{1}$ est localement 1 -H-convexe, en ce sens que chaque point de $\bar{\Omega}$ possède une base de voisinages relatifs à $\bar{\Omega}$ d'adhérences 1-H-convexes.

Dans [CC2], l'équation $\bar{\partial}$ est résolue dans les classes de jets Gevrey d'un compact 1-H-convexe. La démonstration s'adapte sans problème à toute classe de Carleman associée à une suite fortement régulière. Compte tenu de l'exemple (ii) qui précède, on obtient le résultat local suivant:

Proposition $2.4[\mathrm{CC} 2]$. Soient $M$ une suite fortement régulière et $\Omega$ un ouvert borné pseudoconvexe à bord $C^{1}$ dans $\mathbb{C}^{n}$. Avec les notations de 2.1 , on a l'exactitude du complexe

$$
0 \longrightarrow A_{M} \longrightarrow C_{M} \stackrel{\bar{\partial}}{\longrightarrow} C_{M}^{(0,1)} \stackrel{\bar{\partial}}{\longrightarrow} \ldots \stackrel{\bar{\partial}}{\longrightarrow} C_{M}^{(0, n)} \longrightarrow 0 .
$$


Il en résulte classiquement ([G], D5 et D8) que l'on a $H^{q}\left(\bar{\Omega}, A_{M}\right)=H_{\bar{\partial}}^{q}\left(\Gamma\left(\bar{\Omega}, C_{M}^{(0, *)}\right)\right)$ pour $q \geq 1$. En appliquant de nouveau [CC2], on en déduit le résultat global:

Proposition 2.5 [CC2]. Soient $M$ une suite fortement régulière et $\Omega$ un ouvert borné pseudoconvexe à bord $C^{1}$ dans $\mathbb{C}^{n}$, dont l'adhérence est 1-H-convexe. On a alors

$$
H^{q}\left(\bar{\Omega}, A_{M}\right)=0 \quad \text { pour } \quad q \geq 1 .
$$

\section{3. Énoncé des résultats}

3.1. Hypothèses. On considère un ouvert $\Omega$ pseudoconvexe borné dans $\mathbb{C}^{n}$, à bord de classe $C^{1}$. On suppose en outre, dans tout ce qui suit, que l'on a

$$
H^{q}\left(\bar{\Omega}, A_{M}\right)=0 \quad \text { pour } \quad q \geq 1 .
$$

D'après 2.5 , ceci est par exemple vrai dès que $\bar{\Omega}$ est 1 -H-convexe.

On donne $\theta$ une fonction fortement admissible et $M$ une suite fortement régulière.

Soient $p$ un entier avec $1 \leq p \leq n-1$ et $v_{1}, \ldots, v_{p}$ des fonctions de $A_{M}(\bar{\Omega})$, prolongées en éléments de $C_{M}\left(\mathbb{C}^{n}\right)$ (voir 2.2). On pose

$$
\tilde{X}=\left\{z \in \mathbb{C}^{n}: v_{1}(z)=\ldots=v_{p}(z)=0\right\} \quad \text { et } \quad X=\tilde{X} \cap \bar{\Omega} .
$$

On suppose que $\tilde{X}$ rencontre $\partial \Omega$; en particulier on a $X \neq \emptyset$.

On note enfin $\left(v_{1}, \ldots, v_{p}\right) C_{M}(\bar{\Omega})$ (resp. $\left.\left(v_{1}, \ldots, v_{p}\right) A_{M}(\bar{\Omega})\right)$ l'idéal engendré par les $v_{j}$ $\operatorname{sur} C_{M}(\bar{\Omega})\left(\operatorname{resp} . A_{M}(\bar{\Omega})\right)$.

On considère la liste d'hypothèses suivante. Ces hypothèses serviront à tour de rôle dans les différents résultats établis.
$\left(\mathcal{H}_{1}\right) \quad$ Les $v_{j}$ appartiennent à $A_{M}(\bar{\Omega})$.
$\left(\mathcal{H}_{2}\right) \quad$ Les $v_{j}$ appartiennent à $A_{M^{-}}(\bar{\Omega})$.
$\left(\mathcal{H}_{3}\right) \quad$ On a $\partial v_{1} \wedge \ldots \wedge \partial v_{p} \neq 0$ sur $X \cap \partial \Omega$.
$\left(\mathcal{H}_{4}\right) \quad$ Les ensembles $\overline{\tilde{X} \backslash \bar{\Omega}}$ et $\bar{\Omega}$ sont $\theta$-situés.
$\left(\mathcal{H}_{5}\right) \quad$ L'intérieur de $X$ relatif à $\tilde{X}$ est dense dans $X$.

On a alors les énoncés ci-dessous. Les preuves feront l'objet des $\S 4$ et $\S 5$ de ce travail.

THÉORÈme 3.2. Sous les hypothèses $\left(\mathcal{H}_{1}\right)$ et $\left(\mathcal{H}_{3}\right)$, on a

$$
A_{M}(\bar{\Omega}) \cap\left(v_{1}, \ldots, v_{p}\right) C_{M}(\bar{\Omega})=\left(v_{1}, \ldots, v_{p}\right) A_{M}(\bar{\Omega}) .
$$

ThÉORÈme 3.3 (division). Sous les hypothèses $\left(\mathcal{H}_{2}\right),\left(\mathcal{H}_{3}\right),\left(\mathcal{H}_{4}\right),\left(\mathcal{H}_{5}\right)$, soit $f$ une fonction de $A_{M^{-}}(\bar{\Omega})$ telle que l'on ait $\left.f\right|_{X}=0$. Alors $f$ appartient $\grave{a}\left(v_{1}, \ldots, v_{p}\right) A_{M}(\bar{\Omega})$.

ThÉORÈme 3.4 (extension). Sous les hypothèses $\left(\mathcal{H}_{2}\right),\left(\mathcal{H}_{3}\right),\left(\mathcal{H}_{4}\right)$, soit $f$ une fonction de $A_{M^{-}}(X)$. Alors il existe une fonction $g$ de $A_{M}(\bar{\Omega})$ telle que l'on ait $\left.g\right|_{X}=f$.

3.5. Remarques sur les hypothèses $\left(\mathcal{H}_{j}\right)$

(i) Les conditions $\left(\mathcal{H}_{1}\right)$ ou $\left(\mathcal{H}_{2}\right)$ sont vérifiées automatiquement lorsque les $v_{j}$ sont des fonctions holomorphes au voisinage de $\bar{\Omega}$.

(ii) À l'aide des propriétés des fonctions admissibles, on peut s'assurer, en s'inspirant du $§ 0$ de [A2], que $\left(\mathcal{H}_{4}\right)$ ne dépend pas du choix du prolongement à $\mathbb{C}^{n} \operatorname{des} v_{j}$. 
(iii) Lorsque l'ordre de contact entre $\tilde{X}$ et $\partial \Omega$ en chaque point $\zeta$ de $\tilde{X} \cap \partial \Omega$ est au plus égal à $m\left(m \in \mathbb{N}^{*}\right)$, c'est à dire lorsque l'on a $\liminf _{z \longrightarrow \zeta, z \in \tilde{X}}|z-\zeta|^{-m} d(z, \partial \Omega)>0$, il est facile de vérifier que $\left(\mathcal{H}_{4}\right)$ est satisfaite avec $\theta(t)=t^{m}$. La réciproque est fausse excepté dans le cas $m=1, \theta=$ id, où $\left(\mathcal{H}_{4}\right)$ a lieu si et seulement si $X$ est transverse à $\partial \Omega$.

(iv) Dans le cas où $X$ est transverse à $\partial \Omega$, la condition $\left(\mathcal{H}_{5}\right)$ est automatiquement vérifiée.

3.6. Remarques sur les conclusions des théorèmes $3.2,3.3,3.4$.

(i) Lorsque $X$ est transverse à $\partial \Omega$, les théorèmes 3.3 et 3.4 sont sans perte de régularité puisque l'on a alors $\theta=\mathrm{id}, M=M^{-}$.

(ii) Lorsque les $v_{j}$ sont holomorphes au voisinage de $\bar{\Omega}$, la conclusion de 3.2 est à comparer à celle du théorème établi par $\mathrm{A}$. Nagel pour $A^{\infty}(\bar{\Omega})$ ([N], théorème 3.2$)$. Le théorème de Nagel ne requiert cependant pas l'hypothèse $\left(\mathcal{H}_{3}\right)$.

\section{Résultats locaux}

4.1. Notation. On note $\mathcal{I}:=\left(v_{1}, \ldots, v_{p}\right) A_{M}$ et $\mathcal{J}:=\left(v_{1}, \ldots, v_{p}\right) C_{M}$ les faisceaux engendrés respectivement sur $A_{M}$ et $C_{M}$ par les $v_{j}$. Comme conséquence de l'existence de partitions de l'unité de classe $C_{M}$, on a évidemment

$$
\Gamma(\bar{\Omega}, \mathcal{J})=\left(v_{1}, \ldots, v_{p}\right) C_{M}(\bar{\Omega}) .
$$

4.2. Coordonnées locales. L'hypothèse $\left(\mathcal{H}_{3}\right)$ implique que $\tilde{X}$ est une variété lisse au voisinage de $\partial \Omega$ et pour tout point $\zeta$ de $X \cap \partial \Omega$, il existe un voisinage $U$ de $\zeta$ et un $(n-p)$-uple $u$ de fonctions sur $U$ telles que $(u, v):=\left(u_{1}, \ldots, u_{n-p}, v_{1}, \ldots, v_{p}\right)$ forme un système de coordonnées d'origine $\zeta$, appartenant à la classe $C_{M^{-}}$lorsque $\left(\mathcal{H}_{2}\right)$ est vérifiée, $C_{M}$ dans le cas général $\left(\mathcal{H}_{1}\right.$ ), et holomorphes dans $\Omega \cap U$ (voir [D] pour la version $C_{M}$ du théorème des fonctions implicites utilisée ici).

Soit $r$ une fonction définissante pour $\Omega$. On pourra, quitte à renuméroter les $v_{j}$, supposer que l'on a $\partial r \wedge \partial v_{2} \wedge \ldots \wedge \partial v_{p} \neq 0$ dans $U$, c'est à dire que $v_{2}, \ldots, v_{p}$ sont des coordonnées tangentielles (lorsque la coordonnée $v_{1}$ est aussi tangentielle, $\tilde{X}$ est transverse à $\partial \Omega$ au voisinage de $\zeta$ ).

Pour $j=0, \ldots, p$, on notera $X_{j}=\left\{(u, v) \in \mathbb{C}^{n}: v_{j+1}=\ldots=v_{p}=0\right\}$ (on a $X_{0}=\tilde{X}$ et $\left.X_{p}=\mathbb{C}^{n}\right)$. Pour toute partie $Y$ de $\mathbb{C}^{n}$, on pourra identifier $X_{j} \cap Y$ avec sa projection sur $\mathbb{C}^{n-p+j}$.

Dans toute la suite, on utilisera sans le mentionner la stabilité de la régularité Carleman par composition (donc par passage aux coordonnées $(u, v)$ ), voir [D].

Proposition 4.3 (extension locale). On se place dans les hypothèses $\left(\mathcal{H}_{2}\right),\left(\mathcal{H}_{3}\right)$, $\left(\mathcal{H}_{4}\right)$. Soient $\zeta$ un point de $X \cap \partial \Omega$ et $f$ un germe en $\zeta$ de fonction de $A_{M^{-}}(X)$, c'est-àdire un germe de $C_{M^{-}, \zeta}$ qui est $\bar{\partial}$-plat sur $X$. Alors il existe un germe $g$ dans $A_{M, \zeta}$ tel que l'on ait $\left.g\right|_{\tilde{X}}=f$.

Dans cet énoncé et les suivants, les conditions de restriction ou de $\bar{\partial}$-platitude sont bien sûr sous-entendues au voisinage de $\zeta$. Ici, le fait que $g$ coïncide avec $f$ non seulement 
sur $X$, mais même sur $\tilde{X}$ (les germes étant prolongés au voisinage de $\zeta$ ), jouera un rôle important en 5.5.

Pr e u v e. On suit la ligne directrice du théorème 2.1 de [A1] en contrôlant précisément les pertes de régularité. On utilise les coordonnées locales $(u, v)$ dans un voisinage $U$ de $\zeta$. D'après 2.2 , on peut étendre le germe $f$ en une fonction de $C_{M^{-}}\left(\mathbb{C}^{n}\right)$, que l'on notera encore $f$. Pour $u \in \mathbb{C}^{n-p}$, on pose $g_{0}(u)=f(u, 0, \ldots, 0)$ et on considère l'extension triviale de $g_{0}$ à $\mathbb{C}^{n-p+1}$, donnée par

$$
f_{1}\left(u, v_{1}\right)=g_{0}(u),
$$

de sorte que l'on a $f_{1} \in C_{M^{-}}\left(\mathbb{C}^{n-p+1}\right)$,

$$
\left.f_{1}\right|_{\tilde{X} \cap U}=f
$$

et enfin, par hypothèse sur $f$,

$$
\bar{\partial} f_{1} \quad \text { est plat sur } \quad X \cap U .
$$

À partir de $\left(\mathcal{H}_{4}\right)$ et des définitions, il est facile de vérifier que $\overline{\tilde{X} \backslash\left(X_{1} \cap \bar{\Omega}\right)}$ et $X_{1} \cap \bar{\Omega}$ sont $\theta$-situés au voisinage de 0 dans $\mathbb{C}^{n-p+1}$. Compte tenu de (4.3.2), de $1.6-1.8$ et du théorème 2.4 de [Th1], il existe une $(0,1)$-forme $\omega_{1}$ de classe $C_{M}$ dans $\mathbb{C}^{n-p+1}$ telle que l'on ait, quitte à rétrécir $U$,

$$
\begin{gathered}
\omega_{1}=\bar{\partial} f_{1} \text { dans } \quad X_{1} \cap \bar{\Omega} \cap U, \\
\omega_{1} \quad \text { est plate sur } \tilde{X} \cap U .
\end{gathered}
$$

De (4.3.4), on déduit que l'on a, pour tout multi-indice $J$, tout entier $k$ et tout $\left(u, v_{1}\right)$ de $X_{1} \cap U$,

$$
\left|D^{J} \omega_{1}\left(u, v_{1}\right)\right| \leq C^{j+k+1}(j+k) ! M_{j+k}\left|v_{1}\right|^{k} / k !,
$$

où $C$ est une constante convenable. On considère alors la $(0,1)$-forme $\eta_{1}:=v_{1}^{-1} \omega_{1}$, qui est de classe $C^{\infty} \operatorname{sur}\left(X_{1} \backslash \tilde{X}\right) \cap U$. Pour tout multi-indice $L$, on développe $D^{L} \eta_{1}$ à l'aide de la formule de Leibniz et on estime chaque terme $\left(D^{I} v_{1}^{-1}\right)\left(D^{J} \omega_{1}\right)$ apparaissant dans le développement avec $I+J=L$, en utilisant (4.3.5) avec $k=i+2$. Compte tenu de l'estimation triviale $\left|D^{I} v_{1}^{-1}\right| \leq i !\left|v_{1}\right|^{-(i+1)}$ et de la propriété (1.1.2) des suites fortement régulières, on obtient sans difficulté

$$
\left|D^{L} \eta_{1}\left(u, v_{1}\right)\right| \leq C^{l+1} l ! M_{l}\left|v_{1}\right|
$$

pour tout $\left(u, v_{1}\right)$ de $\left(X_{1} \backslash \tilde{X}\right) \cap U$, quitte à augmenter $C$.

Il s'ensuit que $\eta_{1}$ se prolonge en une $(0,1)$-forme de classe $C_{M}$ sur $X_{1}$, plate sur $\tilde{X} \cap U$. Par (4.3.3), on a aussi $\bar{\partial} \eta_{1}=0$ sur $X_{1} \cap \bar{\Omega} \cap U$.

On remarque maintenant que $X_{1} \cap \Omega \cap U$ s'identifie à un ouvert de $\mathbb{C}^{n-p+1}$ à bord de classe $C^{1}$, pseudoconvexe, au voisinage de 0 : c'est une conséquence immédiate du fait que les coordonnées $v_{j}$ sont tangentielles pour $j \geq 2$. On peut appliquer à cet ouvert la proposition 2.4. Quitte à restreindre $U$, il existe donc une fonction $h_{1}$ de classe $C_{M}$ satisfaisant $\bar{\partial} h_{1}=\eta_{1}$ dans $X_{1} \cap \bar{\Omega} \cap U$. D'après $2.2, h_{1}$ peut évidemment être supposée de classe $C_{M}$ à support compact dans $\mathbb{C}^{n-p+1}$.

On pose alors $g_{1}=f_{1}-v_{1} h_{1}$. Clairement, $g_{1}$ est de classe $C_{M}$ dans $\mathbb{C}^{n-p+1}$, on a $\bar{\partial} g_{1}=0$ sur $X_{1} \cap \bar{\Omega} \cap U$ et $\left.g_{1}\right|_{\tilde{X} \cap U}=\left.f_{1}\right|_{\tilde{X} \cap U}=f$ compte tenu de (4.3.1). 
En particulier, pour $p=1$, le germe de $g_{1}$ en $\zeta$ réalise l'extension souhaitée.

Pour $p \geq 2$, on considère $f_{2}$ définie par extension triviale de $g_{1}$ à $\mathbb{C}^{n-p+2}$, c'est-à-dire par

$$
f_{2}\left(u, v_{1}, v_{2}\right)=g_{1}\left(u, v_{1}\right) .
$$

La fonction $f_{2}$ est de classe $C_{M}$ sur $\mathbb{C}^{n-p+2}$ et on a manifestement

$$
\left.f_{2}\right|_{\tilde{X} \cap U}=f .
$$

On a enfin

$$
f_{2} \quad \text { est } \bar{\partial} \text {-plate sur } \quad X_{1} \cap \bar{\Omega} \cap U .
$$

Par ailleurs, la coordonnée $v_{2}$ est tangentielle, donc $X_{1}$ et $X_{2} \cap \partial \Omega$ sont transverses, et les ensembles $\overline{X_{1} \backslash\left(X_{2} \cap \bar{\Omega}\right)}$ et $X_{2} \cap \bar{\Omega}$ sont $i d$-situés, au voisinage de 0 . En utilisant encore [Th1], théorème 2.4 , et $(4.3 .8)$, il existe une $(0,1)$-forme $\omega_{2}$ de classe $C_{M}$ (comme $f_{2}$ ) dans $\mathbb{C}^{n-p+2}$ telle que l'on ait

$$
\begin{gathered}
\omega_{2}=\bar{\partial} f_{2} \quad \text { dans } \quad X_{2} \cap \bar{\Omega} \cap U, \\
\omega_{2} \quad \text { est plate sur } \quad X_{1} \cap U,
\end{gathered}
$$

quitte à réduire $U$.

De là, on montre comme précédemment que $\eta_{2}:=v_{2}^{-1} \omega_{2}$ est de classe $C_{M}$ dans $X_{2}$, plate sur $X_{1}$; on résoud $\bar{\partial} h_{2}=\eta_{2}$ dans $X_{2} \cap \Omega \cap U$ et on pose $g_{2}=f_{2}-v_{2} h_{2}$. Dans le cas $p=2$, compte tenu de (4.3.7), le germe de $g_{2}$ en $\zeta$ réalise l'extension annoncée.

Pour $p \geq 3$, on itère la construction précédente en remarquant que, puisque les coordonnées $v_{3}, \ldots, v_{p}$ sont tangentielles, les ensembles $X_{j}$ et $X_{j+1} \cap \partial \Omega$ seront, à chaque fois, transverses au voisinage de 0 : on conservera donc la régularité $C_{M}$ à chaque étape, contrairement à ce qui se passait dans la construction de $g_{1}$ (passage de $C_{M^{-}}$à $C_{M}$ ).

Proposition 4.4 (division locale). On se place dans les hypothèses $\left(\mathcal{H}_{2}\right),\left(\mathcal{H}_{3}\right),\left(\mathcal{H}_{4}\right)$ et $\left(\mathcal{H}_{5}\right)$. Soit $\zeta$ un point de $X \cap \partial \Omega$ et soit $f$ un germe de $A_{M^{-}, \zeta}$ tel que l'on ait $\left.f\right|_{X}=0$. Alors $f$ appartient $\grave{a} \mathcal{I}_{\zeta}$.

Preuve. Il s'agit de trouver des germes $g_{1}, \ldots, g_{p}$ dans $A_{M, \zeta}$ tels que l'on ait

$$
f=v_{1} g_{1}+\ldots+v_{p} g_{p} .
$$

Dans les coordonnées locales $(u, v)$, le germe $f$ étant supposé prolongé en une fonction de classe $C_{M^{-}}$sur $\mathbb{C}^{n}$ comme indiqué en 2.2 , on a $f(u, 0)=0$ pour $(u, 0) \in X \cap U$. D'après $\left(\mathcal{H}_{5}\right)$, il en résulte que toutes les dérivées de $u \longrightarrow f(u, 0)$ sont également nulles pour $(u, 0) \in X \cap U$. Aussi, si on pose $\varphi_{1}(u, v)=f\left(u, v_{1}, 0, \ldots, 0\right)$ et $\tilde{\varphi}_{1}\left(u, v_{1}\right)=\varphi_{1}\left(u, v_{1}\right)-$ $f(u, 0)$, les jets de classe $C_{M^{-}}$induits par $\varphi_{1}$ sur $E_{1}:=X_{1} \cap \bar{\Omega} \cap U$ (resp. $\tilde{\varphi}_{1}$ sur $\left.E_{2}:=\overline{\left(\tilde{X} \backslash\left(X_{1} \cap \bar{\Omega}\right)\right)} \cap U\right)$ coïncident sur $E_{1} \cap E_{2}$ (on a $E_{1} \cap E_{2} \subset X$ ). En appliquant, compte tenu de $\left(\mathcal{H}_{4}\right)$ et de 1.6-1.8, le théorème 2.4 de [Th1], on obtient une fonction $F_{1}$ de $C_{M}\left(\mathbb{C}^{n-p+1}\right)$ qui coïncide avec $\varphi_{1}$ sur $E_{1}\left(\operatorname{resp} . \tilde{\varphi}_{1}\right.$ sur $\left.E_{2}\right)$, quitte à rétrécir $U$. On en déduit en particulier

$$
\left.F_{1}\right|_{X_{1} \cap \bar{\Omega} \cap U}=f
$$

ainsi que

$$
\left.F_{1}\right|_{\tilde{X} \cap U}=0,
$$


c'est à dire $F_{1}(u, 0)=0$ pour $u$ voisin de 0 dans $\mathbb{C}^{n-p}$. Par suite, quitte à réduire $U$, on a, pour tout $\left(u, v_{1}\right)$ de $X_{1} \cap U$,

$$
F_{1}\left(u, v_{1}\right)=v_{1} G_{1}\left(u, v_{1}\right)+\bar{v}_{1} H_{1}\left(u, v_{1}\right)
$$

avec

$$
G_{1}\left(u, v_{1}\right)=\int_{0}^{1} \frac{\partial F_{1}}{\partial v_{1}}\left(u, t v_{1}\right) d t \quad \text { et } \quad H_{1}\left(u, v_{1}\right)=\int_{0}^{1} \frac{\partial F_{1}}{\partial \bar{v}_{1}}\left(u, t v_{1}\right) d t
$$

Pour $\left(u, v_{1}\right) \in X_{1} \cap U$, si on pose $f_{1}\left(u, v_{1}\right)=v_{1}^{-1} F_{1}\left(u, v_{1}\right)$, on a

$$
f_{1}\left(u, v_{1}\right)=G_{1}\left(u, v_{1}\right)+I_{1}\left(u, v_{1}\right)
$$

avec $I_{1}\left(u, v_{1}\right)=v_{1}^{-1} K_{1}\left(u, v_{1}\right)$ et $K_{1}\left(u, v_{1}\right)=\bar{v}_{1} H_{1}\left(u, v_{1}\right)$. On a vu dans la preuve de 4.3 que $X_{1} \cap \Omega$ s'identifie à un ouvert de $\mathbb{C}^{n-p+1}$, pseudoconvexe à bord $C^{1}$ au voisinage de 0 . On va montrer que $f_{1}$ définit une fonction de classe $A_{M}$ dans cet ouvert. Pour cela, on étudie séparément les termes $I_{1}$, puis $G_{1}$, de (4.4.5).

Pour tout multi-indice $L$ et tout $\left(u, v_{1}\right)$ de $X_{1} \cap U$, on a d'abord, par (4.4.4), la majoration $\left|D^{L} H_{1}\left(u, v_{1}\right)\right| \leq \operatorname{Sup}_{0 \leq t \leq 1}\left|\left(D^{L} \frac{\partial F_{1}}{\partial \bar{v}_{1}}\right)\left(u, t v_{1}\right)\right|$. Or, d'après (4.4.1) et l'holomorphie de $f$, la fonction $\partial F_{1} / \partial \bar{v}_{1}$ est plate sur $X_{1} \cap \bar{\Omega} \cap U$. On en déduit aisément

$$
\left|D^{L} H_{1}\left(u, v_{1}\right)\right| \leq \operatorname{Sup}_{0 \leq t \leq 1}\left(C^{l+k+1}(l+k) ! M_{l+k} \operatorname{dist}\left(\left(u, t v_{1}\right), X_{1} \cap \bar{\Omega} \cap U\right)^{k} / k !\right),
$$

pour tout multi-indice $L$, tout entier $k$ et tout $\left(u, v_{1}\right)$ de $X_{1} \cap \bar{\Omega} \cap U, C$ désignant une constante convenable. Or, pour $\left(u, v_{1}\right) \in X_{1} \cap \bar{\Omega} \cap U$ et $t \in[0,1]$, on a $\operatorname{dist}\left(\left(u, t v_{1}\right), \bar{\Omega} \cap U\right) \leq$ $\left|\left(u, t v_{1}\right)-\left(u, v_{1}\right)\right| \leq\left|v_{1}\right|$. Par (4.4.6) et par définition de $K_{1}$, il vient donc

$$
\left|D^{L} K_{1}\left(u, v_{1}\right)\right| \leq C^{l+k+1}(l+k) ! M_{l+k}\left|v_{1}\right|^{k} / k !,
$$

quitte à augmenter $C$. De là, on tire comme dans la preuve de 4.3 (régularité de $v_{1}^{-1} \omega_{1}$ à partir de (4.3.5)) que $I_{1}$ se prolonge en une fonction de classe $C_{M}$ sur $X_{1} \cap \bar{\Omega} \cap U$, plate sur $X \cap U$.

On vérifie ensuite sans difficulté, à partir de (4.4.1) et de l'holomorphie de $f$, que $G_{1}$ est de classe $C_{M}$ sur $X_{1} \cap \bar{\Omega} \cap U, \bar{\partial}$-plate sur $X \cap U$.

Ainsi, $f_{1}$ est de classe $C_{M}$ sur $X_{1} \cap \bar{\Omega} \cap U, \bar{\partial}$-plate sur $X \cap U$. En outre, elle est clairement holomorphe sur $\left(X_{1} \backslash \tilde{X}\right) \cap \Omega \cap U$, où elle coïncide avec $v_{1}^{-1} f$. On en déduit bien le résultat annoncé précédemment.

À présent, comme $X_{1}$ est transverse à $\partial \Omega$, il existe, d'après 4.3 et quitte à réduire encore $U$, une fonction $g_{1}$ de classe $A_{M}$ dans $\Omega \cap U$ et satisfaisant $\left.g_{1}\right|_{X_{1} \cap \bar{\Omega} \cap U}=f_{1}$. Ceci prouve la proposition pour $p=1$, puisqu'on a alors $f=v_{1} g_{1}$.

Dans le cas $p \geq 2$, on considère la fonction $f-v_{1} g_{1}$ : c'est un germe de $A_{M, \zeta}$, nul sur $X_{1}$ par construction. Suivant l'idée de [A1], théorème 3.1, on répète alors la démarche précédente en construisant $F_{2}$ telle que l'on ait

$$
\left.F_{2}\right|_{X_{2} \cap \bar{\Omega} \cap U}=f-v_{1} g_{1},\left.\quad F_{2}\right|_{X_{1} \cap U}=0,
$$

de façon à construire $g_{2}$ comme extension de $v_{2}^{-1} F_{2}$, et ainsi de suite. On itère ce processus en notant que les constructions successives se font maintenant sans perte de régularité puisque les $X_{j}$ sont transverses à $\partial \Omega$. 
Les lemmes qui suivent serviront au paragraphe 5 .

LEMME 4.5. On se place dans les hypothèses $\left(\mathcal{H}_{1}\right),\left(\mathcal{H}_{3}\right)$. Alors la suite

$$
0 \longrightarrow \mathcal{I} \longrightarrow \mathcal{J} \stackrel{\bar{\partial}}{\longrightarrow} \mathcal{K} \longrightarrow 0
$$

où $\mathcal{K}$ désigne le faisceau image de $\mathcal{J}$ par $\bar{\partial}$, est exacte.

Preuve. Le lemme revient à montrer que si $\zeta$ est un point de $\bar{\Omega}$ et $f$ un germe de la forme

$$
f=v_{1} f_{1}+\ldots+v_{p} f_{p}
$$

avec $f_{j} \in C_{M, \zeta}$ pour $j=1, \ldots, p$, satisfaisant $\bar{\partial} f=0$, alors on peut trouver des germes $g_{j}$ dans $A_{M, \zeta}(1 \leq j \leq p)$ tels que l'on ait $f=v_{1} g_{1}+\ldots+v_{p} g_{p}$.

Lorsque $\zeta$ est un point de $\bar{\Omega} \backslash X$, il existe un indice $i$ tel que $v_{i}$ ne s'annule pas au voisinage de $\zeta$. Le germe de $v_{i}$ en $\zeta$ est alors inversible dans $A_{M, \zeta}$ (voir par exemple [D]) et on a $f=v_{i} g_{i}$ où $g_{i}:=f_{i}+v_{i}^{-1} \sum_{j \neq i} v_{j} f_{j}$ a les propriétés requises.

Lorsque $\zeta$ est un point de $X \cap \Omega$, on remarque d'abord que l'on a $A_{M, \zeta}=\mathcal{O}_{\zeta}$ et $C_{M, \zeta} \subset C_{\zeta}^{\infty}$ : la propriété résulte alors de la $\mathcal{O}$-platitude de $C^{\infty}$, voir [M].

Lorsque $\zeta$ est un point de $X \cap \partial \Omega$, on peut supposer que $f_{1}$ est de classe $C_{M}$ sur $\mathbb{C}^{n}$ et on pose, dans les coordonnées locales $(u, v)$,

$$
\tilde{f}_{1}(u, v)=f_{1}\left(u, v_{1}, 0, \ldots, 0\right) .
$$

Par (4.5.1), on a donc $v_{1} \tilde{f}_{1}\left(u, v_{1}, 0, \ldots, 0\right)=f\left(u, v_{1}, 0, \ldots, 0\right)$ pour $\left(u, v_{1}\right) \in X_{1} \cap \Omega \cap U$. On sait que $X_{1} \cap \Omega \cap U$ s'identifie à un ouvert de $\mathbb{C}^{n-p+1}$ et il résulte alors de cette égalité que $\tilde{f}_{1}$ est $\bar{\partial}$-plate sur $X_{1} \cap \Omega \cap U$.

En appliquant la proposition 4.3 à $\Omega, X_{1}, \tilde{f}_{1}$, donc dans le cas transverse (sans perte de régularité), on obtient un germe $g_{1}$ de $A_{M, \zeta}$ tel que l'on ait $\left.g_{1}\right|_{X_{1} \cap \Omega \cap U}=\tilde{f}_{1}$, quitte à rétrécir $U$.

On considère alors, pour $(u, v) \in \Omega \cap U$, la fonction $h(u, v):=f(u, v)-v_{1} g_{1}(u, v)$. Elle définit un germe de $A_{M, \zeta}$ et s'annule sur $X_{1}$ par construction de $g_{1}$. L'application à $\Omega, X_{1}$ et $h$ de la proposition 4.4, encore une fois dans le cas transverse, assure que l'on a $h(u, v)=\sum_{j=2}^{p} v_{j} g_{j}(u, v)$ où les $g_{j}$ sont des germes de $A_{M, \zeta}$. Ceci prouve le lemme.

Lemme 4.6. On se place dans les hypothèses $\left(\mathcal{H}_{1}\right),\left(\mathcal{H}_{3}\right)$. Soient $\zeta$ un point de $X$ et $f$ un germe de $A_{M, \zeta}$ tel que l'on ait $\left.f\right|_{\tilde{X}}=0$. Alors $f$ appartient à $\mathcal{I}_{\zeta}$.

Preuve. Lorsque $\zeta$ est un point de $X \cap \Omega$ vérifiant $\partial v_{1} \wedge \ldots \wedge \partial v_{p}(\zeta) \neq 0$, le résultat est standard puisque l'on a un système de coordonnées holomorphes $\left(u_{1}, \ldots, u_{n-p}, v_{1}, \ldots, v_{p}\right)$ au voisinage de $\zeta$. Les points $\zeta$ de $X \cap \Omega$ tels que l'on ait $\partial v_{1} \wedge \ldots \wedge \partial v_{p}(\zeta)=0$ sont en nombre fini en vertu de $\left(\mathcal{H}_{3}\right)$ et de propriétés élémentaires des ensembles analytiques. On règle le cas de ces points via la proposition 5.8 de [GS], par exemple. Enfin, lorsque $\zeta$ est un point de $X \cap \partial \Omega$, on pose $F_{1}\left(u, v_{1}\right)=f\left(u, v_{1}, 0, \ldots, 0\right)$. De là, on peut reprendre directement la preuve de 4.4 à partir de (4.4.1)-(4.4.2), puisque $f$ est maintenant supposée d'emblée nulle sur $\tilde{X}$ et non seulement sur $X$ comme dans 4.4 . 
5. Globalisation. On considère les faisceaux $\mathcal{I}$ et $\mathcal{J}$ définis en 4.1 et on note $\mathcal{R}$ le faisceau des relations des $v_{j}$ sur $A_{M}$.

LEMmE 5.1. Sous les hypothèses $\left(\mathcal{H}_{1}\right)$ et $\left(\mathcal{H}_{3}\right)$, on a $H^{q}(\bar{\Omega}, \mathcal{I})=0$ et $H^{q}(\bar{\Omega}, \mathcal{R})=0$ pour $q \geq 1$.

Preuve. Comme dans [A1] ou [GS], il s'agit de vérifier une propriété de cohérence globale. Soient $e_{1}, \ldots, e_{p}$ les sections canoniques de $A_{M}^{p}:=\Lambda^{1} A_{M}^{p}$. On considère le complexe de Koszul

$$
0 \longrightarrow \Lambda^{p} A_{M}^{p} \stackrel{\sigma_{p}}{\longrightarrow} \Lambda^{p-1} A_{M}^{p} \longrightarrow \ldots \longrightarrow \Lambda^{1} A_{M}^{p} \stackrel{\sigma_{1}}{\longrightarrow} \mathcal{I} \longrightarrow 0,
$$

où les $\sigma_{k}: \Lambda^{k} A_{M}^{p} \longrightarrow \Lambda^{k-1} A_{M}^{p}$ sont définis récursivement par $\sigma_{1}\left(e_{j}\right)=v_{j}$ pour $1 \leq j \leq p$ et $\sigma_{k}\left(e^{I} \wedge e_{j}\right)=v_{j} e^{I}-\sigma_{k-1}\left(e^{I}\right) \wedge e_{j}$ pour $2 \leq k \leq p, I=\left(i_{1}, \ldots, i_{k-1}\right)$ avec $1 \leq i_{1}<\ldots$ $\ldots<i_{k-1}<j \leq p$ et $e^{I}=e_{i_{1}} \wedge \ldots \wedge e_{i_{k-1}}$. On justifie d'abord que le complexe (5.1.1) est exact en tout point $\zeta$ de $V \cap \bar{\Omega}$, où $V$ est un voisinage convenable de $\partial \Omega$. Pour cela, il suffit de choisir $V$ tel que l'on ait $\partial v_{1} \wedge \ldots \wedge \partial v_{p} \neq 0$ sur $X \cap V$ : en effet, pour $\zeta \in X \cap V \cap \Omega$, le résultat est standard comme conséquence du fait que $A_{M, \zeta}$ coïncide avec $\mathcal{O}_{\zeta}$ et que $\left(u_{1}, \ldots, u_{n-p}, v_{1}, \ldots, v_{p}\right)$ est un système de coordonnées holomorphes au voisinage de $\zeta$. Dans le cas d'un point $\zeta$ de $X \cap \partial \Omega$, on reprend la preuve d'E. Amar [A1] dans le cadre $A_{M}$ au lieu de $A^{\infty}$, et en s'assurant que la propriété d'extension locale 4.3, utilisée au lieu du théorème 2.1 de [A1], n'intervient que dans le cas transverse, donc sans perte de régularité. Enfin, lorsque $\zeta$ est dans $\bar{\Omega} \cap V \backslash X$, on conclut également comme [A1], en utilisant que l'une des $v_{i}$ ne s'annule pas au voisinage de $\zeta$ et est donc une unité dans $A_{M, \zeta}$.

Comme conséquence, (5.1.1) est une résolution libre de $\mathcal{I}$, globale sur $V \cap \bar{\Omega}$.

Par ailleurs, il est classique que $\left.\mathcal{I}\right|_{\Omega}$ est $\mathcal{O}$-cohérent et

$$
H^{q}(\Omega, \mathcal{I})=0 \quad \text { pour } \quad q \geq 1
$$

On conclut alors que l'on a $H^{q}(\bar{\Omega}, \mathcal{I})=0$ en appliquant la proposition 6.1 de [GS] avec $X=\bar{\Omega}, O=\Omega, A=A_{M}, \mathcal{F}=\mathcal{I}$, compte tenu de (5.1.2) et de l'hypothèse $H^{q}\left(\bar{\Omega}, A_{M}\right)=0$ pour $q \geq 1$.

Les arguments précédents permettent également d'établir $H^{q}(\bar{\Omega}, \mathcal{R})=0$, puisque $\mathcal{R}$ est par définition le noyau de $\sigma_{1}$.

LEMmE 5.2. Sous les hypothèses $\left(\mathcal{H}_{1}\right)$ et $\left(\mathcal{H}_{3}\right)$, on a $\Gamma(\bar{\Omega}, \mathcal{I})=\left(v_{1}, \ldots, v_{p}\right) A_{M}(\bar{\Omega})$.

Preuve. En reprenant les notations de la preuve de 5.1, la suite

$$
0 \longrightarrow \mathcal{R} \longrightarrow A_{M}^{p} \stackrel{\sigma_{1}}{\longrightarrow} \mathcal{I} \longrightarrow 0
$$

est exacte. Compte tenu de l'annulation de $H^{1}(\bar{\Omega}, \mathcal{R})$ en 5.1 , on en déduit l'exactitude de la suite

$$
0 \longrightarrow \Gamma(\bar{\Omega}, \mathcal{R}) \longrightarrow \Gamma\left(\bar{\Omega}, A_{M}^{p}\right) \stackrel{\sigma_{1}^{*}}{\longrightarrow} \Gamma(\bar{\Omega}, \mathcal{I}) \longrightarrow 0
$$

avec $\sigma_{1}^{*}\left(f_{1}, \ldots, f_{p}\right)=\sum_{j=1}^{p} v_{j} f_{j}([\mathrm{G}], \mathrm{D} 2)$. Le résultat s'ensuit.

Lemme 5.3. Sous les hypothèses $\left(\mathcal{H}_{1}\right)$ et $\left(\mathcal{H}_{3}\right)$, soit $f$ un élément de $\Gamma(\bar{\Omega}, \mathcal{J})$ tel que l'on ait $\bar{\partial} f=0$. Alors $f$ appartient à $\Gamma(\bar{\Omega}, \mathcal{I})$. 
Preuve. L'exactitude de la suite

$$
0 \longrightarrow \Gamma(\bar{\Omega}, \mathcal{I}) \longrightarrow \Gamma(\bar{\Omega}, \mathcal{J}) \stackrel{\bar{\partial}}{\longrightarrow} \Gamma(\bar{\Omega}, \mathcal{K})
$$

résulte aussitôt $([\mathrm{G}], \mathrm{A} 10) \mathrm{du}$ lemme 4.5.

5.4. Preuve du théorème 3.2. Immédiate par 5.2, 5.3 et (4.1.1).

5.5. Preuve du théorème 3.3. Pour tout point $\zeta$ de $\bar{\Omega}$, il existe un voisinage $U_{\zeta}$ de $\zeta$ dans $\mathbb{C}^{n}$ et des germes $g_{j}^{\zeta}$ de $A_{M, \zeta}$ tels que l'on ait $f=\sum_{j=1}^{p} v_{j} g_{j}^{\zeta}$ dans $U_{\zeta}$ (Pour $\zeta \in X \cap \partial \Omega$, c'est la proposition 4.4, pour $\zeta \in X \cap \Omega$ le résultat s'obtient en invoquant les mêmes arguments que dans la preuve de 4.6, enfin pour $\zeta \in \bar{\Omega} \backslash X$, c'est encore une conséquence de l'inversibilité de l'un des $v_{j}$ dans $\left.A_{M, \zeta}\right)$. À l'aide d'une partition de l'unité de classe $C_{M}$ subordonnée à un recouvrement de $\bar{\Omega}$ par un nombre fini de $U_{\zeta}$, on en déduit que $f$ est dans l'idéal $\left(v_{1}, \ldots, v_{p}\right) C_{M}(\bar{\Omega})$ et on conclut avec 3.2.

5.6. Preuve du théorème 3.4. Par 4.3 on construit un recouvrement fini de $\bar{\Omega}$ par des ouverts $U_{i}$ de $\mathbb{C}^{n}$ et des fonctions $g_{i}$ de classe $A_{M}$ sur $\bar{\Omega} \cap U_{i}$ satisfaisant $\left.g_{i}\right|_{\tilde{X}}=f$. Soit $\zeta$ un point de $U_{i} \cap U_{j}$; alors $g_{i}-g_{j}$ appartient à $A_{M, \zeta}$ et s'annule sur $\tilde{X}$. D'après 4.6, on a $g_{i}-g_{j} \in \mathcal{I}_{\zeta}$. Ainsi les extensions locales $g_{i}$ de $f$ définissent un élément $\tilde{g}$ de $\Gamma\left(\bar{\Omega}, A_{M} / \mathcal{I}\right)$. Par ailleurs, l'exactitude de la suite

$$
0 \longrightarrow \Gamma(\bar{\Omega}, \mathcal{I}) \longrightarrow \Gamma\left(\bar{\Omega}, A_{M}\right) \longrightarrow \Gamma\left(\bar{\Omega}, A_{M} / \mathcal{I}\right) \longrightarrow 0
$$

est une conséquence standard $([\mathrm{G}], \mathrm{D} 2)$ de l'annulation de $H^{1}(\bar{\Omega}, \mathcal{I})$ en 5.1. Aussi $\tilde{g}$ se relève en un élément $g$ de $A_{M}(\bar{\Omega})$ dont le germe en tout point $\zeta$ de $\bar{\Omega}$ coïncide avec celui de $f$ modulo $\mathcal{I}_{\zeta}$, d'où l'on tire $\left.g\right|_{X}=f$.

Remarque 5.7. Tous les résultats décrits dans ce travail concernent le cas où la codimension $p$ de $\tilde{X}$ est au plus $n-1$. Pour $p=n, \tilde{X}$ est réduit à un point et les conclusions de $3.2,3.3,3.4$ restent vraies sous les seules hypothèses $\left(\mathcal{H}_{1}\right)$ et $\left(\mathcal{H}_{3}\right)$, avec $M^{-}=M$. En effet l'extension est alors triviale, la division locale se réduit au lemme 4.6; enfin les lemmes 5.1 à 5.3 s'adaptent aisément, ce qui permet de globaliser.

\section{Références}

[A1] E. Amar, Cohomologie complexe et applications, J. London Math. Soc. (2) 29 (1984), 127-140.

[A2] E. Amar, Non-division dans $A^{\infty}(\bar{\Omega})$, Math. Z. 188 (1985), 493-511.

[DBT] P. de Bartolomeis \& G. Tomassini, Finitely generated ideals in $A^{\infty}(\bar{D})$, Adv. in Math. 46 (1982), 162-170.

[BBMT] J. Bonet, R. W. Braun, R. Meise \& B. A. Taylor, Whitney's extension theorem for nonquasianalytic functions, Studia Math. 99 (1991), 156-184.

[B] J. Bruna, An extension theorem of Whitney type for non quasi-analytic classes of functions, J. London Math. Soc. (2) 22 (1980), 495-505.

[CC1] J. Chaumat \& A.-M. Chollet, Théorème de Whitney dans des classes ultradifférentiables, C. R. Acad. Sci. Paris Sér. I Math. 315 (1992), 901-906. 
[CC2] J. Chaumat \& A.-M. Chollet, Noyaux pour résoudre l'équation $\bar{\partial}$ dans des classes ultradifférentiables sur des compacts irréguliers de $\mathbb{C}^{n}$, in: Several Complex Variables (Stockholm 1987/1988), Math. Notes 38, Princeton Univ. Press, Princeton, 1993, 205-226.

[D] E. M. Dynkin, Pseudoanalytic extension of smooth functions. The uniform scale, Amer. Math. Soc. Transl. 115 (1980), 33-58.

[GS] R. Gay \& A. Sebbar, Division et extension dans l'algèbre $A^{\infty}(\bar{\Omega})$ d'un ouvert pseudoconvexe à bord lisse de $\mathbb{C}^{n}$, Math. Z. 189 (1985), 421-447.

[G] R. C. Gunning, Introduction to Holomorphic Functions of Several Variables, vol. III : Homological Theory, Wadsworth \& Brooks/Cole, Monterey, 1990.

[M] B. Malgrange, Ideals of Differentiable Functions, Oxford University Press, Bombay, 1966.

[N] A. Nagel, On algebras of holomorphic functions with $C^{\infty}$ boundary values, Duke Math. J. 41 (1974), 527-535.

[Th1] V. Thilliez, Prolongement dans des classes ultradifférentiables et propriétés de régularité des compacts de $\mathbb{R}^{n}$, Ann. Polon. Math. 63 (1996), 71-88.

[Th2] V. Thilliez, Sur les fonctions composées ultradifférentiables, J. Math. Pures Appl. (9) 76 (1997), 499-524. 